ARTICLE

Received 17 Feb 2015 | Accepted 31 May 2015 | Published 9 Jul 2015

DOI: $10.1038 /$ ncomms 8680

OPEN

\title{
Two routes to senescence revealed by real-time analysis of telomerase-negative single lineages
}

Zhou $\mathrm{Xu}^{1,2}$, Emilie Fallet ${ }^{1,2}$, Camille Paoletti ${ }^{3}$, Steffen Fehrmann ${ }^{3}$, Gilles Charvin ${ }^{3} \&$ Maria Teresa Teixeira ${ }^{1,2}$

In eukaryotes, telomeres cap chromosome ends to maintain genomic stability. Failure to maintain telomeres leads to their progressive erosion and eventually triggers replicative senescence, a pathway that protects against unrestricted cell proliferation. However, the mechanisms underlying the variability and dynamics of this pathway are still elusive. Here we use a microfluidics-based live-cell imaging assay to investigate replicative senescence in individual Saccharomyces cerevisiae cell lineages following telomerase inactivation. We characterize two mechanistically distinct routes to senescence. Most lineages undergo an abrupt and irreversible switch from a replicative to an arrested state, consistent with telomeres reaching a critically short length. In contrast, other lineages experience frequent and stochastic reversible arrests, consistent with the repair of accidental telomere damage by Pol32, a subunit of polymerase $\delta$ required for break-induced replication and for post-senescence survival. Thus, at the single-cell level, replicative senescence comprises both deterministic cell fates and chaotic cell division dynamics.

\footnotetext{
${ }^{1}$ Centre National de la Recherche Scientifique, UMR8226, Laboratoire de Biologie Moléculaire et Cellulaire des Eucaryotes, Institut de Biologie Physico-Chimique, 75005 Paris, France. ${ }^{2}$ Sorbonne Universités, UPMC University Paris 06, UMR8226, Laboratoire de Biologie Moléculaire et Cellulaire des Eucaryotes, Institut de Biologie Physico-Chimique, 75005 Paris, France. ${ }^{3}$ Institut de Génétique et de Biologie Moléculaire et Cellulaire, 1 rue Laurent Fries, Illkirch, 67400, France. Correspondence and requests for materials should be addressed to M.T.T. (email: teresa.teixeira@ibpc.fr).
} 
T he reverse transcriptase telomerase counteracts the loss of telomere sequences during eukaryotic DNA replication. In human somatic cells, which generally lack telomerase, telomere shortening eventually causes replicative senescence and thus serves as a mechanism to limit cell division and prevent uncontrolled proliferation, as, for example, in cancer ${ }^{1,2}$. Current models suggest that when one or several telomeres reach a critical length, they lose the protective cap and expose naked DNA, thereby activating a DNA damage checkpoint pathway that results in cell-cycle arrest ${ }^{3,4}$. In mutant Saccharomyces cerevisiae lacking telomerase, gradual telomere shortening eventually leads to a similar replicative senescent state $e^{5,6}$. Some rare cells may overcome senescence by elongating telomeres through either reactivation of telomerase or alternative recombination-based mechanisms ${ }^{7,8}$. In mammals, such variants are precursors of cancer cells. Therefore, elucidating the mechanisms underlying the establishment of senescence may shed light on the relationship between telomere dysfunction and carcinogenesis 9 .

Replicative senescence is an intrinsically heterogeneous process. In S. cerevisiae, cell-to-cell variations in cell-cycle duration and generation number are influenced by many factors, including the initial telomere length distribution (more precisely, the initial length of the shortest telomere $e^{10,11}$ ), the stochasticity of the telomere-shortening mechanism ${ }^{12}$ and other less wellcharacterized events ${ }^{13,14}$. This heterogeneity is supported by analyses of single senescent colonies ${ }^{5,14,15}$; however, methods that allow precise time-resolved analysis of individual cell lineages over multiple generations are still lacking. Such methods would undoubtedly provide a more accurate understanding of the molecular events controlling senescence.

Here we developed a microfluidics device to analyse consecutive cell divisions from telomerase inactivation to cell death and characterize the transition into replicative senescence. We found that the replicative dynamics of individual lineages contrasts sharply with the classical view of a progressive loss of proliferation potential during senescence, and instead reflects an abrupt lengthening of the cell cycle over the course of one or two cell cycles. Unexpectedly, we also discovered that a substantial proportion of lineages did not behave in a manner consistent with canonical telomere-shortening-driven signalling, but instead exhibited intermittent periods of cell-cycle arrest at DNA damage checkpoints followed by resumption of normal cell cycles before finally entering terminal senescence. This behaviour could not be explained by classical models of gradual telomere attrition. Cells with this phenotype persist only at low frequency in bulk cultures, making them undetectable in conventional population-averaged assays. However, they could provide a basis for further genomic alterations with dramatic consequences, such as cancer emergence.

\section{Results}

A microfluidics device to track senescent cell lineages. To characterize and address the cause of cell-to-cell variations in senescence, we have developed a single-cell imaging methodology based on a microfluidic device that allows individual cell lineages to be tracked with high spatial and temporal resolution (Fig. 1a,b $)^{16}$. The microfluidics chip includes two main channels for the flow of medium and an array of central chambers that house the cells of interest (Fig. 1b). Yeast cells are loaded into the small chambers, where they divide and invade microcavities (Fig. 1b,c and Supplementary Movie 1). The cell reaching the tip of the microcavity can then be monitored using phase contrast and fluorescence microscopy. In $S$. cerevisiae, cell division is asymmetric and mother cells can produce only a limited number of daughters. This phenomenon, termed mother cell ageing, is observed regardless of telomerase inactivation ${ }^{16,17}$. To investigate telomerase-dependent replicative senescence in single dividing cells without interference from mother cell ageing, the tracked cell should have a replicative age low enough to prevent the appearance of ageing phenotypes (typically $<5$ divisions, Supplementary Fig. 1a,b and Supplementary Movie 1). We therefore used the haploid W303 strain background, which displays an essentially bipolar budding pattern because of a bud4 mutation ${ }^{18}$. This ensured that the cell at the tip of the microcavity was frequently replaced by its daughter cells. To avoid tracking cells that were ultimately ejected from the microcavity, we selected an individual cell at the point of death (or termination of the experiment) and retrospectively tracked the preceding cell divisions to recreate its entire lineage (see Methods). With this set-up, we were able to monitor single-cell lineages for $>70$ divisions under physiological conditions (Fig. 1d, Supplementary Fig. 1c and Supplementary Movie 1).

To ensure complete temporal control over telomerase inactivation, we used a doxycycline-repressible TLC1 gene encoding telomerase template RNA (TetO2-TLC1). In the absence of doxycycline, lineages with active telomerase showed no sign of mortality (Fig. 1c,d). However, after telomerase repression by the addition of doxycycline, TetO2-TLC1 cells underwent a limited and highly heterogeneous number of divisions before cell death $(37 \pm 12$ (median \pm s.d.); coefficient of variation $(\mathrm{CV}))=0.32$; Fig. 2a-c and Supplementary Movie 2 and 3). To determine whether the initial telomere length distribution contributed to this variability, we analysed clonal populations (in which the initial cell starts with a unique telomere length distribution) of a telomerase-inactive strain (described below). This strain displayed significantly smaller variations in division number before lysis $(\mathrm{CV}=0.11$ and 0.15 for two clones; Supplementary Fig. 2a,b), suggesting that the heterogeneous response to telomerase loss observed with TetO2-TLC1 cells was predominantly because of interclonal variations in the initial telomere length distribution.

The proliferative capacity of individual TetO2-TLC1 cell lineages observed here is lower than that measured in bulk populations of cells, which undergo $40-80$ population doublings depending on the strain background and initial mean telomere length ${ }^{5,12,19}$. We hypothesized that this apparent discrepancy may be due to competition and selection bias intrinsic to bulk cultures (that is, fitter cells outgrow slow-growing or arrested cells), which is absent in our single-cell analyses. To test this, we performed an in silico competitive growth assay on the basis of the probability of death and cell-cycle duration extracted from all single-lineage data (Supplementary Fig. 3a,b). This simulation quantitatively recapitulated the growth curves observed experimentally in batch cultures of the same strain (Supplementary Fig. 3c), confirming that the larger number of doublings before senescence observed in population growth assays was a consequence of the selection of fit cells.

Two distinct phenotypes upon telomerase removal. Importantly, our single-cell tracking method allowed us to precisely determine the cell-cycle durations of individual lineages, which cannot be determined from population studies. We found that nearly all TetO2-TLC1 lineages displayed one or more particularly long cell cycle leading to cell death (indicated in red in Fig. 2c), at which point up to $70 \%$ of the cells were arrested in G2/ $\mathrm{M}$ (Fig. 2b, $t=85 \mathrm{~h}$ ), consistent with previous observations of senescence arrest ${ }^{14}$. The longer cell cycles were not associated with the older mother cells, ruling out the contribution of mother cell ageing to replicative senescence in this experimental setting (Supplementary Fig. 1b). Strikingly, a significant fraction of lineages showed a distinct phenotype, with successive long cell 


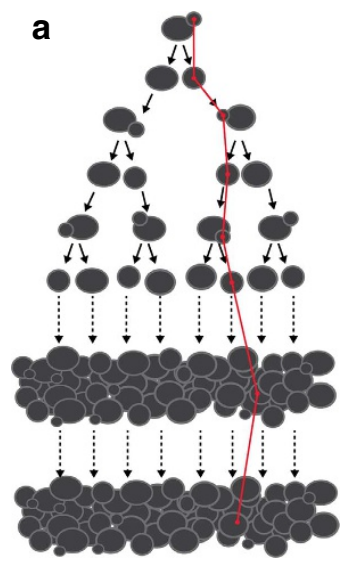

b

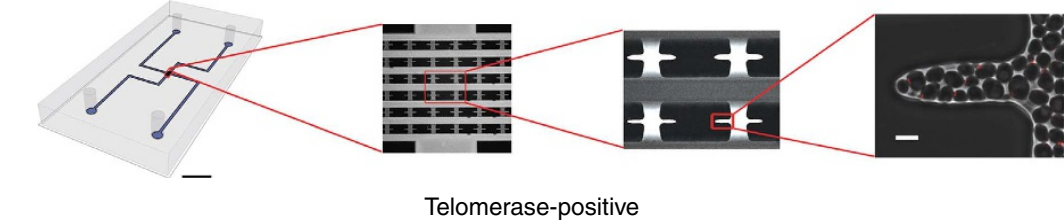

C

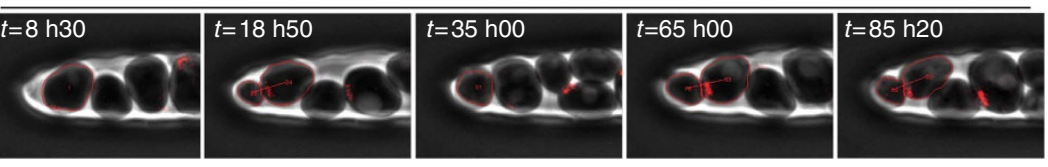

d

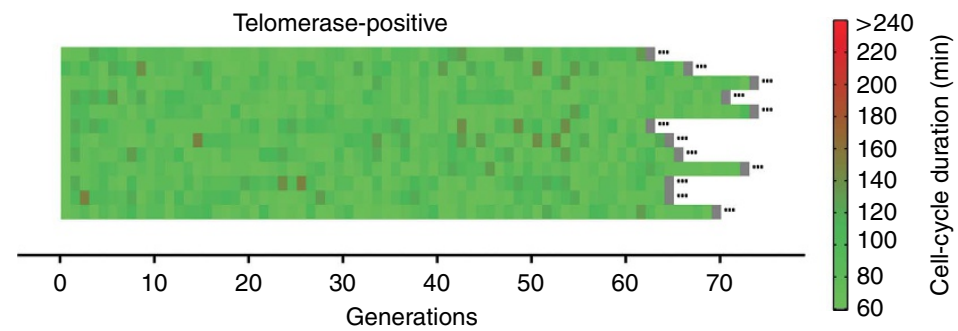

Figure 1 | A microfluidics-based approach to the analysis of single lineages. (a) Schematic representation of single-lineage tracking (in red). Starting from a single cell, we followed the lineage by tracking one of the two cells after each division, regardless of the daughter/mother cell status. (b) Image of the microfluidics chip showing the design of the chambers and microcavities. Scale bars, $5 \mathrm{~mm}$ (black) and $5 \mu \mathrm{m}$ (white). (c) Overlays of sequential phase contrast and fluorescence images of a telomerase-positive cell lineage. The Cdc10-mCherry marker at the bud neck (red) allows monitoring of cell-cycle progression and the mother-daughter relationship. (d) Display of independent wild-type lineages (yT538, $n=12$ ). Each horizontal line represents a single lineage, and each segment is a cell cycle. The ellipsis (...) at the end of each lineage indicates that the cell was still alive at the end of the experiment. Cell-cycle duration is indicated by the colour bar.

a

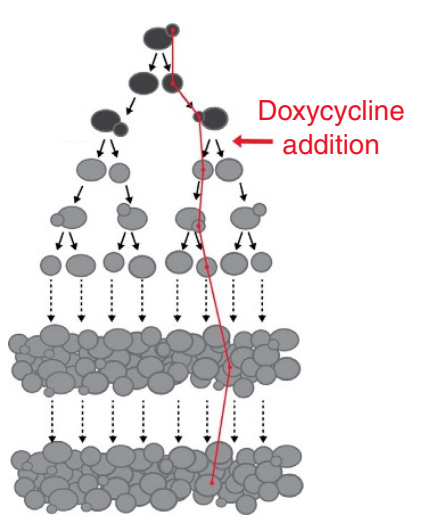

b

\begin{tabular}{c} 
Telomerase- $\begin{array}{l}\text { Doxycycline } \\
\text { positive }\end{array}$ \\
\hline$t=8 \mathrm{~h} 30$
\end{tabular}
$\begin{aligned} & t=18 \mathrm{~h} 50 \\
& \text { addition }\end{aligned}$

C

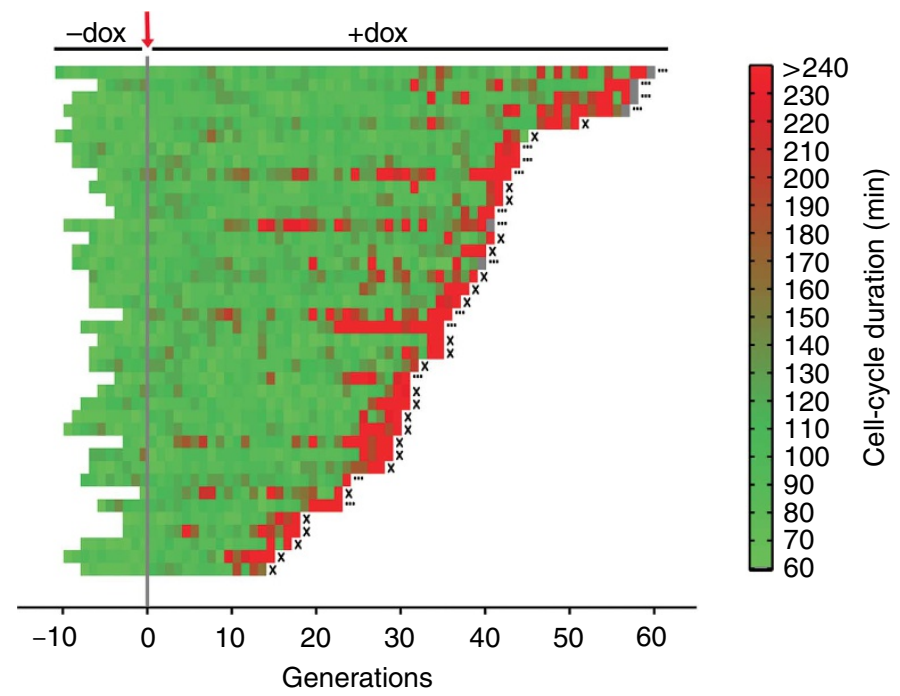

Figure 2 | Telomerase inactivation-induced replicative senescence in single lineages. (a) Schematic representation of senescence-tracking in the TetO2-TLC1 strain (as in Fig. 1a). (b) Sequential phase contrast and fluorescence images (as in Fig. 1c) of a TetO2-TLC1 cell lineage. Addition of doxycyline renders the lineage telomerase-negative. (c) Display of TetO2-TLC1 lineages (yT528, $n=40$ ). Cells were grown in microfluidic chambers for several generations before the addition of doxycycline $\left(30 \mu \mathrm{g} \mathrm{ml}^{-1}\right.$ in the flowing medium) to inactivate telomerase at generation 0 . An ellipsis ( ...) indicates that the cell was alive at the end of the experiment and $X$ indicates cell death. A grey segment indicates that the cell cycle was not complete at the end of the experiment. 


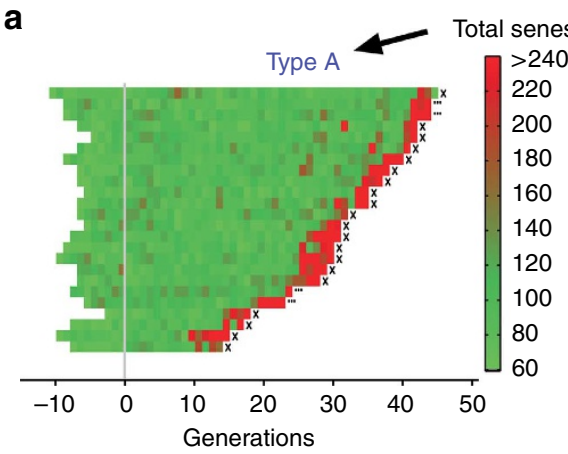

b

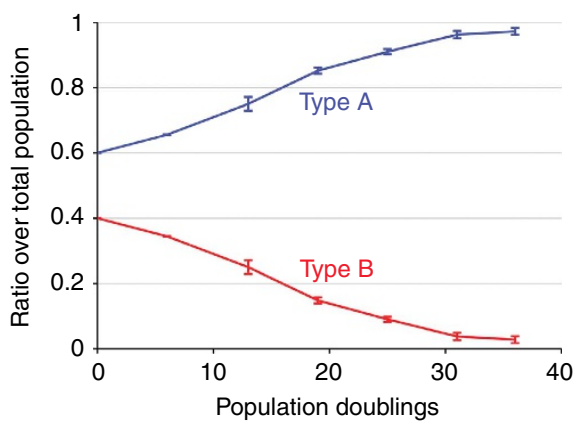

Type B

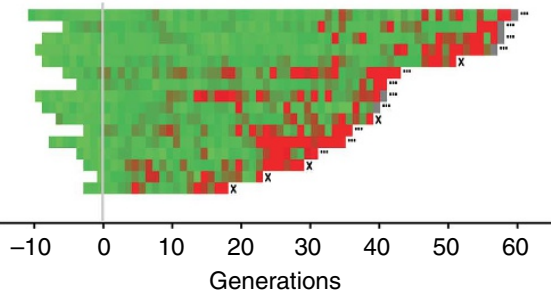

C

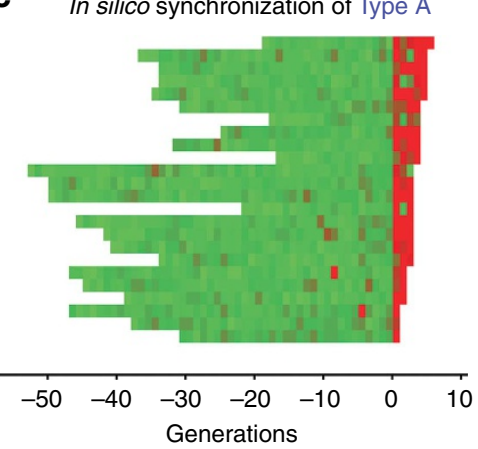

Figure 3 | Identification of two routes to senescence. (a) The senescent lineages shown in Fig. 2c were clustered according to the absence (type A) or presence (type B) of at least two long cell cycles (exceeding the mean +3 s.d. duration of wild-type cell cycles) before the terminal arrests.

(b) Simulation of the competition between type A and type B lineages in a virtual bulk culture. The simulation was limited to $\sim 35$ population doublings because of the lack of experimental data in type A lineages beyond this point. (c) Type A lineages synchronized in silico at the point of transition to replicative senescence, indicated by 0 generation on the $x$ axis.

cycles occurring well before entry into terminal senescence (Fig. 2c). To quantify this, we defined a significantly long cell cycle as having a duration greater than the mean +3 s.d. of the cycles of telomerase-positive cells. We then clustered the lineages according to the absence (type A, $60 \%$ of the lineages) or presence (type B, $40 \%$ of the lineages) of at least two long cell cycles before the terminal successive arrests (Fig. 3a). To evaluate the robustness of this threshold-based clustering, we performed two additional independent clustering analyses on the basis of Gaussian distribution mixture models and obtained nearly identical frequencies of type $\mathrm{A}$ and type $\mathrm{B}$ lineages (Supplementary Fig. 4). To assess the relative competitive potential of type A and B lineages, we simulated their growth in a mixed bulk culture and found that type A lineages rapidly outgrew type B lineages because of their shorter division times (Fig. 3b), even though type B cells had greater median chronological longevity than type A cells $(135(99 ;+\infty)$ and $74(66 ; 77) \mathrm{h}$; median $(95 \%$ confidence interval (CI)), respectively, Supplementary Fig. 5). These data suggest that, whereas type A lineages correspond to the commonly studied 'senescent cells', type B lineages represent a 'cryptic' phenotype of telomerase inactivation. Despite being present at a significant frequency, the type B lineage is virtually invisible in population studies and can only be revealed in single-cell analyses that eliminate competition bias.

Type A lineages displayed up to 40 cell cycles of duration $1.5 \pm 0.3 \mathrm{~h}$ (mean \pm s.d.) before undergoing a sharp transition to one or more long cycles ( $12 \pm 11 \mathrm{~h}$; Fig. $3 \mathrm{c}$ and Supplementary Fig. 6). This abrupt change suggests that a single event and/or a threshold effect triggers senescence arrest, which contrasts with the view that senescence involves a gradual decrease in the growth rate, as proposed by studies based on averaging of individual lineages ${ }^{5,14}$. Moreover, the transition to slower division and arrest is irreversible in type A lineages, indicating that any attempt at telomere repair fails to reverse the senescence signalling state. Type A lineages would thus be consistent with gradual telomere shortening that goes unnoticed by the cell-cycle checkpoints until one or several telomeres reach a critically short length and the cell cycle is irreversibly arrested.

In contrast, the early cell-cycle delays observed in type B lineages are reversible, as indicated by the resumption of normal cycling following one or more precocious long cycles (Fig. 3a). To check whether type B cells are not simply type A cells that have escaped arrest, we compared the time of onset of the first significantly long cell cycle in both cell types and found a significantly earlier onset for type B cells than for type A cells (17.5 \pm 13.4 and $30 \pm 9.8$ divisions, respectively; median \pm s.d., $P=0.03$, unpaired $t$-test). Moreover, the first long cell cycle was clearly a harbinger of death for type A cells but not for type B cells, as indicated by the number of divisions between the first and last long cell cycles $(3.5 \pm 1.4$ and $23 \pm 7.8$ divisions for type A and B cells, respectively). In addition, fewer of the cell-cycle arrests experienced by type B cells occurred in G2/M (48\% compared with $70 \%$ ).

Collectively, these data suggest that the type A and B phenotypes may represent distinct mechanistic responses to telomerase inactivation. Although progressive telomere shortening to a critically short length can account for the behaviour of type A lineages, this model cannot easily account for the precocious cell-cycle delays of type B lineages.

Stochastic telomere defects cause early cell-cycle delays. To gain further insights into the origin of type B early cell-cycle delays, 
we next asked whether they were caused by the loss of a non-canonical telomerase function ${ }^{20}$. For this, we analysed the est2-D670A mutant, which carries a mutation in the reverse transcriptase motif of Est2, the catalytic subunit of telomerase ${ }^{21}$. In the two est2-D670A clones analysed, type $\mathrm{A}$ and type $\mathrm{B}$ lineages were detected in ratios similar to those of the TetO2-TLC1 strain (Supplementary Figs 2a,b and 6d). These data confirm that the long cell cycles observed in type B cells are due to a telomerase elongation defect and not to the loss of a non-canonical function.

The est2-D670A clones, obtained by the spontaneous loss of a complementing plasmid, were selected as single colonies and grown in liquid medium before injection into the microfluidics device. Thus, in these experiments, each lineage started with a single telomerase-negative cell. We concluded that commitment to the type B pathway is not determined by the initial cell or by the initial telomere set but, rather, occurs stochastically. We therefore predicted that a TetO2-TLC1 strain with longer initial telomeres in an otherwise isogenic background would produce type B lineages with a higher frequency because the delay in type A lineage senescence would provide an extended window of time for type B long cycles to occur. Indeed, we observed a much higher proportion of type $B$ lineages in the strain with longer initial telomeres than in the strain with normal telomere length ( $\sim 82 \%$ compared with 40\%; Supplementary Figs 7a, 8 and $6 \mathrm{~d}$ ), although the kinetics of telomere shortening in the two strains were similar (Supplementary Fig. 7b). Interestingly, the strain with longer telomeres did not show detectable telomere breaks or display unusual telomere attrition kinetics. This observation suggests that such events either do not correlate with type B lineage arrests or occur only in a subset of lineages and in an asynchronous manner, making them extremely difficult to detect in population-based assays. Overall, the results of these experiments support the notion that type B cells arise stochastically and further confirm that type A and B lineages arise through distinct mechanisms.

Asymmetry and memory in type B lineages. To further investigate the origin of the cell-cycle delays, we compared the cell-cycle durations of pairs of sister cells born from a single mitotic event. The cell-cycle durations in type A pairs showed a strong correlation (Fig. 4a, correlation coefficient (95\% CI) $r=0.97(0.96 ; 0.98))$, even for the long cycles $(r=0.98(0.93$; 0.99)). These findings are consistent with senescence being signalled by a single critically short telomere following replication by the semiconservative DNA replication machinery and then being transmitted through mitosis to both cells ${ }^{10-12}$. In contrast, a weaker correlation was observed for cell-cycle durations in type
B pairs (Fig. $4 b, r=0.73(0.64 ; 0.80)$ ), particularly for the long cycles $(r=0.61(0.40 ; 0.77))$, further arguing that these cycles occur stochastically. We next asked whether the first precocious long cell cycle dictates the duration of subsequent ones by keeping a 'memory' of the initial duration. For this, we calculated the autocorrelation function of cell-cycle duration in the lineages ${ }^{22}$. Whereas wild-type cells had an autocorrelation function showing no memory of cell-cycle duration over divisions (timescale $\tau=0.6(0.5 ; 0.7)$ divisions), telomerasenegative lineages had a specific cellular memory $(\tau=1.5(1.2 ; 2.0)$ divisions; Fig. 4c). Furthermore, only type B lineages contributed to this autocorrelation $(\tau=3.2(2.5 ; 4.5)$ divisions), whereas type A lineages were more comparable to wild-type cells and exhibited no significant memory $(\tau=1.0(0.9 ; 1.3)$ division). Thus, type B cells are fundamentally imbued with a long-term memory of previous cell-cycle durations. Taken together, our results suggest the transmission through mitosis of a marker that is generated stochastically in the absence of telomerase, signals cell-cycle delay and increases the probability of additional long cell cycles in type $\mathrm{B}$, but not type A, lineages.

DNA damage checkpoint activation in senescence. To determine whether this marker is related to DNA damage, we deleted the major DNA damage checkpoint kinase, Mec1/ATR, which is also involved in signalling short telomeres in the absence of telomerase in budding yeast ${ }^{11,14,15}$. This was performed in a sml1 $\triangle$ background to suppress the lethality of MEC1 deletion ${ }^{23}$. Indeed, MEC1 SML1 deletions abolished senescence-specific 'memory', as indicated by the autocorrelation function of doxycycline-treated TetO2-TLC1 mec1A sml1A cells $(\tau=0.9$ $(0.6 ; 1.4)$ divisions, Fig. 4c). Moreover, while SML1 inactivation suppressed many precocious long cell cycles, loss of Mec1 further reduced the frequency of both precocious and terminal consecutive long cell cycles, which no longer exhibited extended G2/M phases (Fig. 5a and Extended Data Supplementary Fig. 9a,b), indicating that early cell-cycle delays in type B cells indeed stemed from DNA damage. As early cell-cycle delays were not observed in the presence of telomerase elongating activity, these results indicate that the delays are caused by premature telomeric damages sensed by the DNA damage checkpoint pathway.

Impact of Rad51 on cell viability and telomere maintenance. Type B cells are able to resume normal cell cycles after slower divisions, suggesting that following damage and checkpoint activation, cells may undergo adaptation ${ }^{24}$ or recover after the
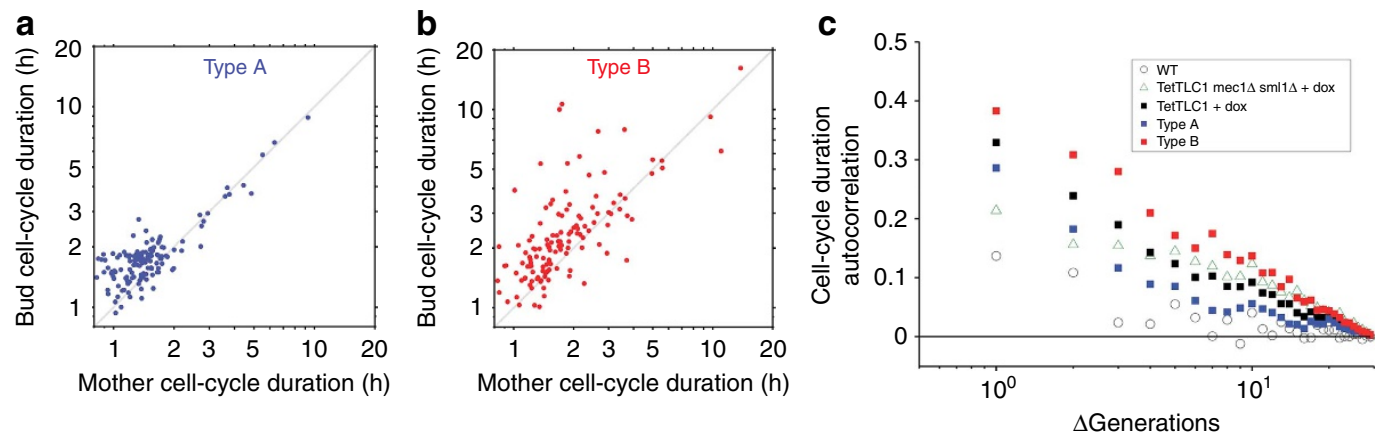

Figure 4 | Transmission of the cell-cycle arrest signal at the mitosis and lineage scale. $(\mathbf{a}, \mathbf{b})$ Scatter plots of cell-cycle duration in pairs of sister cells (mother cell and new daughter) in TetO2-TLC1 (a) type A lineages ( $n=141$ pairs from three independent lineages) and (b) type B lineages ( $n=131$ pairs from three independent lineages). Correlation coefficients $(95 \% \mathrm{Cl})$ between the pairs are $(\mathbf{a}) r=0.97(0.96 ; 0.98)$ and $(\mathbf{b}) r=0.73(0.64 ; 0.80)$.

(c) Autocorrelation functions of cell-cycle durations in the indicated strains treated with or without doxycycline. Telomerase-negative lineages, particularly type B cells, show stronger autocorrelation than telomerase-positive cells. Mec1 deficiency suppresses memory. 
a

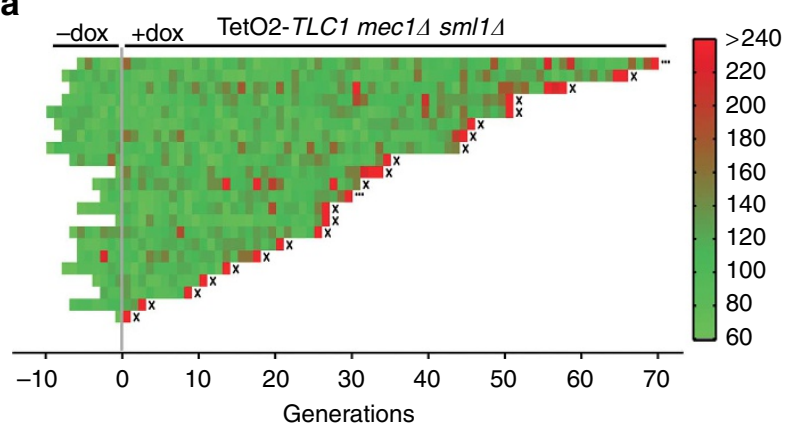

C

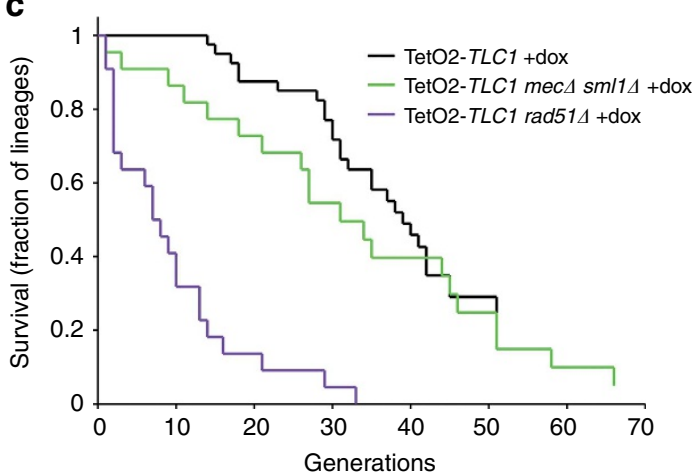

b

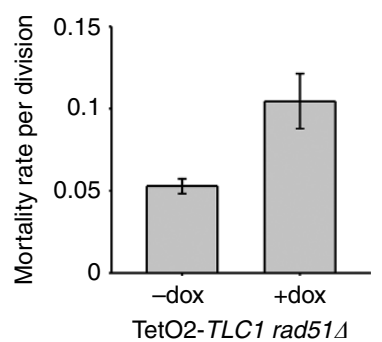

d

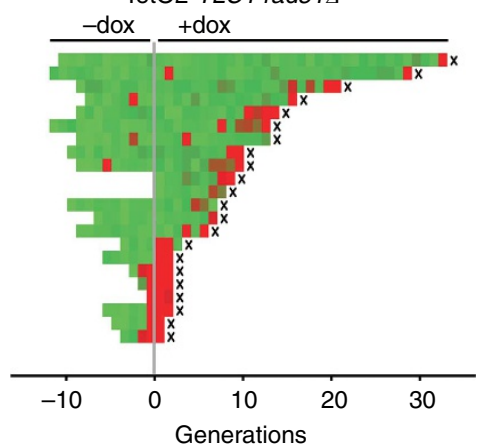

Figure 5 | DNA damage checkpoint and homologous recombination shape senescence dynamics. (a) Analysis of telomerase-negative mec14 sml14 lineages $(y T 598, n=22$ ). (b) Mortality rate per division of RAD51 mutants with or without telomerase activity ( \pm doxycycline). See Supplementary Fig. $9 d$ for calculation of mortality rates. Error bars represent $95 \% \mathrm{Cl}$. (c) Survival curves of the indicated telomerase-inactive strains. (d) Analysis of telomerase-inactivated rad514 lineages (yT641, $n=22$ ).

damage is repaired. We thus evaluated the contribution of homology-directed repair (HDR), which is known to act at telomeres in the absence of telomerase $e^{6,711,25-28}$. Deletion of $R A D 52$, a pivotal HDR factor, caused most lineages to die within 10 divisions in our microfluidics assay, regardless of the presence of active telomerase. This precluded a quantitative analysis of the contribution of Rad52 to senescence. Loss of the recombinase Rad51 also dramatically increased the rate of spontaneous cell death (compare Supplementary Fig. 9c with Fig. 1d), albeit to a lesser extent than Rad52 deficiency. The survival curve of active telomerase-expressing rad514 cells decays exponentially, indicating that they are subject to stochastic death events with a constant mortality rate of $\sim 5 \%$ per division (Fig. $5 \mathrm{~b}$ and Supplementary Fig. 9d). This is probably due to genomic instability ${ }^{29}$ and, notably, only slightly decreases overall growth in bulk liquid culture (Supplementary Fig. 3c). Although Rad51 associates with the shortest telomeres ${ }^{25,30}$ and has genome-wide effects in the presence of telomerase, its contribution to cell viability early after telomerase inactivation remains elusive. Here our single-lineage analyses show that upon telomerase inactivation, most rad51 $\Delta$ lineages died within 10 divisions, at which time telomeres had shortened by only $\sim 30 \mathrm{bp}$ (Fig. $5 c$,d, Supplementary Fig. 7c and Supplementary Movie 4). Inactivation of telomerase in the rad514 mutant increased mortality from $\sim 5$ to $\sim 11 \%$ per division (Fig. 5b,d and Supplementary Fig. 9d), whereas telomerase inactivation in Rad51-positive cells had no effect on mortality during the first 10 divisions (Fig. 5c). Thus, the increase in mortality reveals a synergy between Rad51 and telomerase in the protection of telomeres that is distinct from the genome-wide role of Rad51. Furthermore, the exponential decay in the survival curve indicates that, in cells lacking both Rad51 and telomerase, death is a stochastic and age-independent process. Overall, the analysis of single-cell lineages has allowed us to detect and quantify the constitutive contribution of homologous recombination to telomere protection, which presumably occurs by preventing or rescuing telomere break events, as previously proposed ${ }^{31}$.

Type B lineages are suppressed in the absence of Pol32. Breakinduced replication (BIR) is a HDR pathway in which one end of a broken chromosome can invade a homologous region and prime DNA replication up to the end of the template chromosome. This pathway prevails at telomeres in the absence of telomerase and relies on Pol32, a nonessential subunit of polymerase $\delta$ (refs 32-35; Fig. 6a,b). In the presence of telomerase, POL32 deletion increased the mortality rate to $\sim 0.7 \%$ per division and increased the average cell-cycle duration and variability (108 $\pm 38 \mathrm{~min}$, Fig. $6 \mathrm{~b}$ and Supplementary Fig. 1c). This result suggests that Pol32 promotes efficient cell-cycle progression, consistent with its role in BIR. Cells in which both telomerase and Pol32 were inactivated underwent $17.5 \pm 16$ divisions (median \pm s.d.), indicating an acceleration of replicative senescence, as reported previously ${ }^{19,25}$ (Fig. 6a). This acceleration was not due to differences in telomere length or in telomereshortening kinetics (Supplementary Fig. 7d). We used cluster analysis to separate pol32 $\Delta$ senescent lineages into types A and B, adapting the threshold for significantly long cell cycles (mean +3 s.d. $\approx 220 \mathrm{~min}$ ) to account for the longer average cell-cycle duration observed in the telomerase-positive pol324 cells (Fig. 6c and Supplementary Fig. 1c). Strikingly, the absence of Pol32 reduced the proportion of type B lineages from 40 to $\sim 22 \%$ (Fig. 6c,d). This ratio was probably an overestimation because some of the observed arrests may have occurred regardless of the lack of telomerase (compare Fig. $6 \mathrm{~b}, \mathrm{c}$ ). Indeed, the overall frequency of non-terminal arrests in pol324 
a

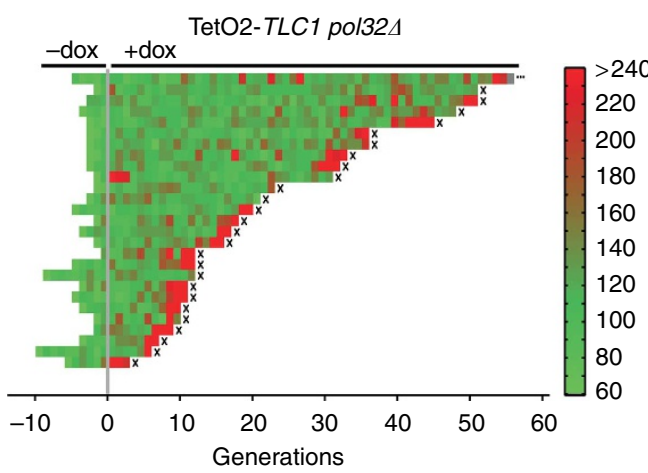

C

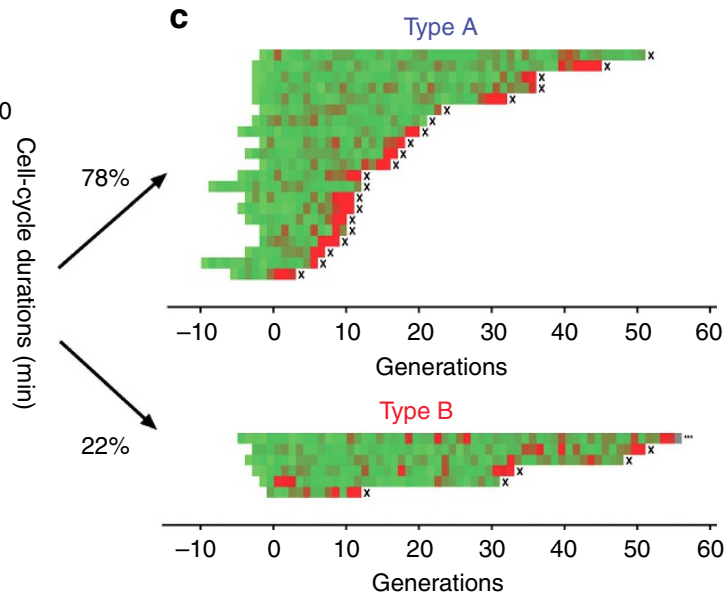

b

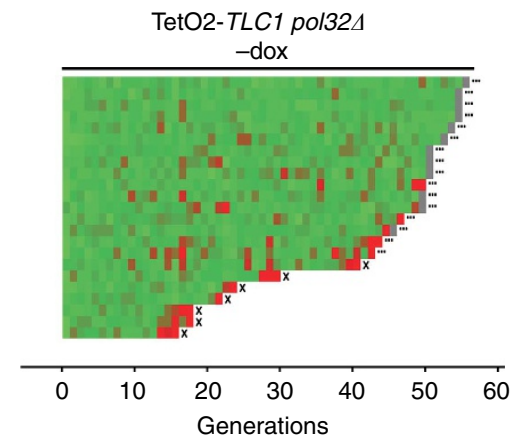

d

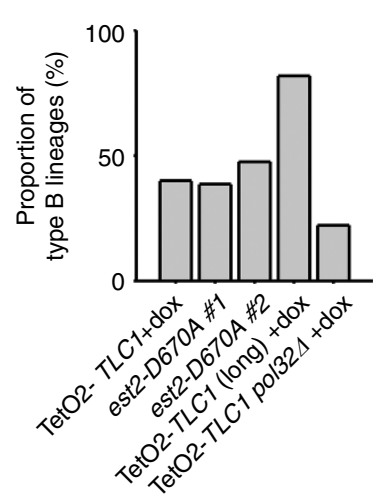

e

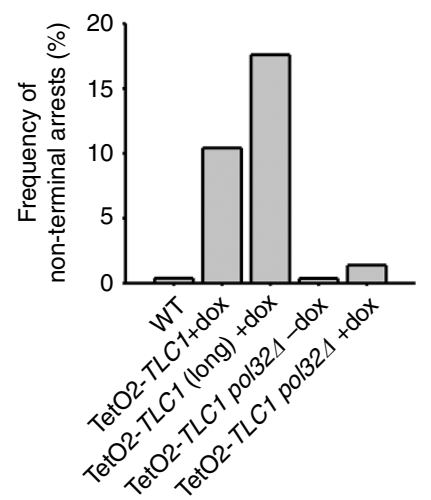

Figure 6 | Pol32 is required for type B lineages. (a) Analysis of telomerase-negative pol324 lineages (yT662, $n=27$ ). (b) Analysis of telomerase-positive pol324 lineages $(y T 662, n=23$ ). (c) Telomerase-negative pol324 lineages were clustered into type A and type B lineages. (d) Quantification of the proportion of type B lineages in the indicated strains. The 'TetO2-TLC1 (long) + dox' strain corresponds to the strain with longer initial telomeres shown in Supplementary Fig. 8 and Supplementary Fig. 7a, lanes 3 and 4, which displayed a higher frequency of type B lineages. (e) Quantification of the frequency of non-terminal arrests in the indicated strains, used as an alternative assessment of the proportion of type $B$ lineages.

telomerase-negative and -positive lineages were $\sim 1.4 \%$ and $\sim 0.4 \%$, respectively (Fig. $6 \mathrm{e}$ ), indicating that $\sim 26 \%(0.4 / 1.4)$ of the non-terminal arrests in the telomerase-negative lineages were due to the deletion of POL32, regardless of the absence of telomerase activity. However, because replicative senescence was accelerated in the pol324 strain, we reasoned that the reduced proportion of type B lineages might reflect the lack of opportunity for precocious arrests rather than a mechanistic suppression of type B lineages. We thus calculated the frequency of non-terminal arrests in telomerase-negative POL32 and pol32 4 strains as an alternative method of assessing type $B$ lineage frequency independently of the length of the lineages. We found that this frequency was $\sim 10 \%$ in telomerase-negative POL32 and $\sim 1.4 \%$ in telomerase-negative pol32 4 , arguing in favour of a genuine suppression of the early arrests occurring in type $B$ lineages (Fig. 6e). Therefore, our data strongly suggest that Pol32 contributes to the formation of type B lineages and that BIR may be involved in the repair and recovery of the precocious damage. In the absence of Pol32-dependent repair, type B-specific damage would lead to cell death, making the type A and B lineages indistinguishable and thus accounting for the observed acceleration of replicative senescence. Nonetheless, we cannot exclude the possibility that Pol32 may also play a minor role in type A lineages; for example, in the few terminal arrests, but the impact of BIR-based repair seems far more important in the type B lineages.

\section{Discussion}

Our single-lineage and time-resolved analysis of senescence has uncovered two distinct phenotypes on telomerase removal. The phenotypes could not have been characterized using conventional population studies, which are based on averages of individual events and favour the selection of the most proliferative lineages. Several lines of evidence demonstrate that the type A and B telomerase-negative lineages occur via biologically distinct routes. First, they follow distinct kinetics to the first cell-cycle delay and to cell death and are separable by modulating the initial telomere length. Second, the early long cell cycles are reversible in type B lineages but not in type A lineages. Third, the DNA damages causing the cell-cycle delays in the two lineages are different both in terms of transmission through mitosis and requirements for Pol32 for repair. Importantly, the behaviour of both type A and type $\mathrm{B}$ lineages is inconsistent with a progressive increase in cell-cycle duration as the telomeres shorten.

In type A lineages, the kinetics of the switch into senescence correlates with the first telomere(s) reaching a critically short length(s), suggesting that this may be the senescence trigger. Likewise, the symmetric transmission of the cell-cycle arrest phenotype in mitosis is reminiscent of segregation of a genetic marker such as telomere length. To verify this, we developed a mathematical model of senescence taking into account the molecular mechanism of telomere shortening ${ }^{12}$ and different hypotheses for the senescence onset signal. We found a perfect 
match between type A experimental data and simulations based on the hypothesis that a single telomere reaching a critically short length triggers an irreversible cell-cycle arrest (Bourgeron et al., unpublished). Furthermore, type A cells undergo a single transition into very long and abnormal cell cycles followed by cell death. Therefore, eroded telomeres seem strongly resistant to repair.

In contrast, type B lineages experience early spontaneous and reversible cell-cycle delays, that often persist in cell lineages, and are inherited in an asymmetrical manner, and are thus inconsistent with gradual telomere erosion. While the molecular mechanisms causing the first arrest in type $B$ lineages remain unclear, the cells seem to keep a memory of this event, which is suppressed by the absence of Mec1 and Sml1. Several non-mutually exclusive hypotheses are consistent with this phenomenon: (i) the telomeres may be in a partially unprotected state that stochastically signals an arrest in subsequent cell cycles, (ii) the cells may undergo adaptation ${ }^{24,36}$, thus proceeding through a few divisions before arresting again because the initial damage has not been repaired, (iii) extrachromosomal markers, such as telomeric ssDNA circles found in postsenescence survivors ${ }^{37}$, may be generated and poorly segregated or (iv) the initial telomere damage may be repaired by a BIR or another recombination mechanism but generates genetic or telomeric instabilities that can promote more telomeric or genome-wide damage. In accordance with this latter scenario, the early delays observed in type B lineages are suppressed by telomerase elongation activity itself and by the absence of Mec1, Sml1 or Pol32, suggesting that they correspond to telomeric DNA damages, repaired by BIR. BIR could subsequently lead to the elongation of the damaged telomeres by $\mathrm{Y}^{\prime}$ subtelomeric element $^{35}$, but also, in combination with a checkpoint deregulation or adaptation, can initiate a cascade of genetic instabilities, shown to occur both in yeast and human cells in some circumstances ${ }^{38-40}$.

The finding that the absence of Rad51 and Rad52 has a strong effect on the viability of all telomerase-negative lineages suggests that these factors may act constitutively to protect telomere replication under conditions of replication stress ${ }^{41,42}$. An alternative, but not mutually exclusive, possibility is that the recombination factors could act by preventing the accumulation of ssDNA and signalling by the shortest telomere $(\mathrm{s})^{25}$. In addition, Rad51 and Rad52 could contribute in a Pol32independent manner ${ }^{19}$ to repair the stochastic telomeric damage in type B lineages in combination with BIR mechanisms. Such damage could be a consequence of the observed replication stress at telomeric repeats, for example $25,31,43-46$.

Taken together, our results are consistent with a model in which telomere replication is continuously protected by HDR pathways and by BIR when rare accidental breaks occur. Type A lineages would avoid such breaks, whereas type B lineages would experience an erratic pattern of cell-cycle arrests that contribute to the heterogeneity of senescence. Because of their altered checkpoint state and their dependence on Pol32, type B lineages may accumulate mutations and experience cascades of genome rearrangements, eventually leading to the emergence of genomic variants $33,38-40,47-49$. Finally, the cryptic status and extended longevity of type B lineages may allow them to re-emerge in longterm cultures and give rise to post-senescent survivors. We speculate that in humans similar type B lineages could well be the precursors of cancer cells in aged tissues.

\section{Methods}

List of strains. All of the strains used in this study had a W303 background and were corrected for the rad5-535 mutation, which is naturally present in the W303 strain. See Supplementary Table 1.
Growth assay. To measure the proliferation potential of telomere-positive and telomere-negative cells, we diluted a log-phase culture of TetO2-TLC1 RAD51 (yT528) or TetO2-TLC1 rad514 (yT641) strains to $\mathrm{OD}_{600 \mathrm{~nm}}=2.3 \times 10^{-5}$ in YPD medium and grew them at $30^{\circ} \mathrm{C}$ for $24 \mathrm{~h}$ until $\mathrm{OD}_{600 \mathrm{~nm}}$ reached 1.5. The cultures were then divided and grown with or without $30 \mu \mathrm{g} \mathrm{ml}^{-1}$ doxycycline to repress TLC1. Each day, the same procedure was applied with an adjustment of the dilution ratio in the telomerase-inactivated cultures in order to reach $0.6<\mathrm{OD}_{600 \mathrm{~nm}}<2$, after $24 \mathrm{~h}$, to account for the loss of proliferation potential. For monitoring the kinetics of telomere shortening, a similar growth assay was performed except that the cultures were diluted daily to $\mathrm{OD}_{600 \mathrm{~nm}}=0.01$ in YPD + doxycycline $\left(30 \mu \mathrm{g} \mathrm{ml}^{-1}\right)$ and the cells were collected after $24 \mathrm{~h}$ of growth.

Terminal restriction fragment Southern blot. Genomic DNA was extracted from exponential growth cultures using a standard phenol:chloroform:isoamyl (25:24:1) purification procedure and ethanol precipitation. Then, $1 \mu \mathrm{g}$ of genomic DNA was digested with XhoI and the products were ethanol-precipitated, resuspended in loading buffer (10 mM Tris pH 8.0, $1 \mathrm{mM}$ EDTA, 5\% glycerol, $0.04 \%$ xylene cyanol FF) and resolved on a $1.2 \%$ agarose gel for $14 \mathrm{~h}$ at $60 \mathrm{~V}$. The gel was then soaked in a denaturation bath $(0.4 \mathrm{M} \mathrm{NaOH}$ and $1 \mathrm{M} \mathrm{NaCl})$ for $30 \mathrm{~min}$ and transferred by capillarity action to a charged nylon membrane (Hybond XL, GE Healthcare). The telomere-specific oligonucleotide probe $\left(5^{\prime}\right.$-GGGTGTGGGTGTGTGT GGTGGG-3 $3^{\prime}$ was ${ }^{32} \mathrm{P}$-labelled at the $5^{\prime}$ terminus with ATP $\left(\gamma_{-}{ }^{32} \mathrm{P}\right)$ and T4 polynucleotide kinase (New England Biolabs). The membrane was hybridized using the Rapid-hyb buffer protocol (GE Healthcare). In brief, the membrane was prehybridized at $42^{\circ} \mathrm{C}$ in Rapid-hyb buffer for $1 \mathrm{~h}$, then the radioactive probe ( $20 \mathrm{pmol}$ ) was added and the incubation was continued for $1 \mathrm{~h}$. The membrane was washed consecutively with $2 \times \mathrm{SSC}, 0.5 \% \mathrm{SDS}\left(42^{\circ} \mathrm{C}\right.$ for $\left.10 \mathrm{~min}\right) ; 2 \times \mathrm{SSC}, 0.1 \%$ SDS $\left(42^{\circ} \mathrm{C}\right.$ for $\left.20 \mathrm{~min}\right)$; and $0.1 \times \mathrm{SSC}, 0.1 \% \operatorname{SDS}\left(20^{\circ} \mathrm{C}\right.$ for $\left.30 \mathrm{~min}\right)$. The membrane was then imaged with a Typhoon FLA 9500 scanner (GE Healthcare)

Telomere elongation. In some experiments (Supplementary Figs 2 and 8), the telomeres were elongated before cells were placed in the device, either to replenish telomere repeats subsequently lost during preculture and sample preparation (Supplementary Fig. 2) or to study the effect of longer telomeres (Supplementary Fig. 8). To reversibly elongate telomeres, we grew cells in rich medium (YPD) containing $5 \%$ ethanol for $\sim 80$ or $\sim 160$ generations, serially diluting every 3 days $^{50}$. The cells were then transferred to YPD agar plates without ethanol, and at this point the mean telomere length was measured with Southern blot analysis, as described above and shown in Supplementary Fig. 7a. To ensure the recovery of cells from ethanol treatment, we grew cells for at least 40 divisions in medium without ethanol before placing them in the microfluidic device.

Microfluidic device. The microfluidic mold was fabricated using standard soft lithography techniques as described ${ }^{16}$. To make the chip, we mixed polydimethylsiloxane (PDMS; Sylgard 184) and curing agent in a 10:1 ratio, degassed it with a vacuum pump for $30 \mathrm{~min}$ and poured it into the mold. The PDMS was cured by baking at $70^{\circ} \mathrm{C}$ for $5 \mathrm{~h}$ and then was carefully removed from the mold. A biopsy puncher (1.5 mm, Harris Unicore) was used to create holes for medium flow. The surfaces of PDMS and a glass coverslip $(24 \times 50 \mathrm{~mm})$ were surface-activated using a plasma cleaner (Diener Electronic, Germany) to covalently bond the two elements. For injection of cells into the device, synthetic complete medium containing $2 \%$ glucose (SD) was filtered using a $0.22-\mu \mathrm{m}$ polyethersulfone filter (Corning) and loaded into the device using a peristaltic pump (IPCN, Ismatec). Cells from a log-phase culture $\left(0.5 \mathrm{OD}_{600}\right)$ were gently injected into the device using a 1-ml syringe. A constant medium flow $\left(28 \mu \mathrm{lmin}{ }^{-1}\right)$ was maintained throughout the experiment. Control experiments validating the flow rate, medium diffusion into the cavities, nutrient uptake and physiological cell growth were performed as previously described ${ }^{16}$. For experiments with strains expressing the TetO2-TLC1 construct, cells were allowed to divide and invade the cavities for $12-24 \mathrm{~h}$ before the medium was switched to SD containing $30 \mu \mathrm{g} \mathrm{ml}^{-1}$ doxycycline. For experiments with the est2-D670A mutant strain (yT639), a clone without the plasmid complementing EST2 deficiency (pVL291 encoding EST2 with a URA3 marker) was selected. Because selection required 20-30 cell divisions, the telomeres were elongated by ethanol treatment before the experiments (see above, Supplementary Figs 2 and $7 \mathrm{a}$ ). To select a single clone, cells were plated on YPD for $24 \mathrm{~h}$ and individual colonies were picked and grown on selecting medium (SD-uracil) to verify plasmid loss (the frequency of spontaneous loss was 5-8\%). After an additional $24 \mathrm{~h}$, a colony without plasmid was precultured overnight in SD medium and injected into the microfluidic device as described above. An aliquot of the same culture was analysed using Southern blot analysis to measure telomere length (Supplementary Fig. 7a).

Time-lapse microscopy. Cells in the microfluidic device were imaged using a fully motorized Axio Observer Z1 inverted microscope (Zeiss), with constant focus maintained with focus stabilization hardware (Definite focus, Zeiss). To minimize phototoxicity, we used light-emitting diode (LED) light sources for both phase contrast and fluorescence images (Colibri 2, Zeiss) with the following parameters: $4.0 \mathrm{~V}-70 \mathrm{~ms}$ for phase contrast and $15 \%$ of maximum intensity $-400 \mathrm{~ms}$ with $2 \times 2$ binning for the $560 \mathrm{~nm}$ LED. The temperature was maintained at $30^{\circ} \mathrm{C}$ 
with a controlled heating unit and an incubation chamber that held the entire microscope base, including the stage and the objectives. Images were acquired every 10 min using AxioVision 4 (Zeiss). All aspects of image acquisition were fully automated and controlled, including temperature, focus, stage position and time-lapse imaging. Images were acquired for $>120 \mathrm{~h}$ in standard experiments and up to $240 \mathrm{~h}$ in the experiment using cells with elongated telomeres.

Image analysis and single-cell lineage tracking. A custom software written in Matlab, phyloCell 2.1, was used to segment and track cells and to assign motherdaughter links ${ }^{16}$. Time-lapse images were exported as high-resolution TIF files and analysed directly with the graphical user interface of phyloCell. To follow cell-cycle progression and determine the mother-daughter relationship, cells expressed a fluorescent Cdc10-mCherry fusion protein that localized to the septin ring from bud formation to cytokinesis. This fluorescent marker was also used to determine the cell-cycle stage based on the morphology and size of the bud. For details about the routines and algorithms implemented in phyloCell, see ref. 16. In contrast to ref. 16, the cell at the tip of the cavity would frequently be replaced by its daughter cell, which was intended in our approach. To efficiently track such lineages, in which we frequently switched focus from a given cell to its daughter cell, the time-lapse images were retrospectively analysed starting from the last image. This avoided tracking of lineages in which the cells were ejected from the microcavity.

Computational and statistical analyses. All computational and statistical analyses were performed in Matlab. The functions developed in this study are available on request. For cluster analysis of telomerase-negative lineages, two independent criteria were used: the number of long cell cycles (greater than the mean +3 s.d. of wild-type cell cycle, which may be different from strain to strain) per lineage and the number of transitions per lineage. A transition was defined as two consecutive cycles with a difference in their duration greater than a threshold $S$. The number of transitions per lineage was calculated for $S=60,70,80,90$ and $100 \mathrm{~min}$ and averaged for each lineage. For each of these two criteria, we created a mixture model with univariate Gaussian distributions and performed a cluster analysis of the mixture, which defined clusters as data points belonging most probably to the same distribution. The gmdistribution class and related methods of the statistics toolbox of Matlab were used for this analysis.

Autocorrelation function. The normalized autocorrelation function $A(k)$ for lag $k$ was calculated according to ${ }^{22}$ :

$$
A(k)=\left\langle\frac{\mathrm{E}_{n}\left[\left(Y_{n}-\bar{Y}\right)\left(Y_{n+k}-\bar{Y}\right)\right]}{\mathrm{E}_{n}\left[\left(Y_{n}-\bar{Y}\right)^{2}\right]}\right\rangle,
$$

where $Y_{n}$ denotes the cell-cycle duration at division $n$ normalized over the wild-type mean for mother or newborn daughter cell accordingly; $\bar{Y}$ denotes the mean of $Y$ over all time points and cells; $\mathrm{E}_{n}[]$ denotes the expected value operator over divisions; and \langle\rangle denotes the average over all cells. The autocorrelation function $A(k)$ measures the similarity between two cell-cycle durations as a function of the time lag $k$ between them. For a given strain or condition, for any $k$, $A(k)$ was averaged over all cell cycles of all lineages. The function was fitted with an exponential function $t \rightarrow e^{-t / \tau}$ to obtain an estimate of the autocorrelation timescale $\tau$ of the process. Typically, $A(k)$ rapidly drops to 0 after several cell cycles, and the timescale $\tau$ of this decrease indicates how fast the information of a cellcycle duration is lost.

Simulation of competitive culture. To compare cell growth in the microfluidic chip and conventional bulk culture, we numerically simulated a virtual culture in which the analysed lineages would be in competition. From the single-lineage data (Fig. 2c), we extracted the probability of death and the cell-cycle duration landscape (Supplementary Fig. 3b) and used them as inputs to simulate competitive growth (that is, the total number of cells in the virtual culture as a function of time). The simulation of a telomerase-negative culture was performed with a custom automaton developed in Matlab. All parameters were directly derived from the independent lineage data (Supplementary Fig. 3b), and no arbitrary external parameters were used. First, for each generation $n$ after telomerase loss, a probability of death $P(n)=\frac{\mathrm{d} s(n)}{\mathrm{d} n}$, where $S$ is the survival curve, was derived from the fraction of total lineages that ended at this generation. Given the discrete nature of the data, $P$ was fitted with a Gaussian function. Second, we calculated a matrix $M(n, p)$ containing the probability that a cell at generation $n$ will divide in $p$ units of time (that is, $10 \mathrm{~min}$, which is the temporal resolution of the time-lapse experiments). The number of rows in $M$ is restricted to the maximum number of generations undergone by a lineage. $M$ is calculated by normalizing the experimental frequency two-dimensional histogram of cells dividing at generation $n$ in $p$ units of time, shown in Supplementary Fig. 3b. The simulation included dilution steps to mimic those required in experimental senescing cultures. The dilution ratio and the number of cells picked to continue the virtual culture had no detectable effect on the result (data not shown). The automaton starts with 1,000 cells, each of which is randomly assigned a cell-cycle duration chosen from $M(1,[1, \max ])$, that is, the first row of $\mathrm{M}$. For each time point, we determine whether a cell has completed its cycle and then test whether this cell, which is at generation $n$, will die, given $P(n)$. If it dies, that cell is removed from the array. If not, it divides into two cells (a new cell is created in the automaton), each of which is assigned a new cell-cycle duration corresponding to the generation $n+1$, chosen from $M(n+1,[1, \max ])$. To smooth the discrete data, we pick $p$ from $M$ and account for the variability of biological processes by adding noise according to the $\mathrm{CV}$ experimentally observed. This simulation is performed over 60 time points, corresponding to $10 \mathrm{~h}$. The total number of cells is recorded. Then, 1,000 cells are randomly picked from this virtual culture and are run in a second round of simulation (dilution). After 13 rounds, all data are collected and converted into a plot, as shown in Supplementary Fig. 3a. For estimation of the fraction of type A and type B cells in a bulk culture (Fig. 3b), two cell-cycle duration matrices $M_{\mathrm{A}}$ and $M_{\mathrm{B}}$ were derived from the two sets of lineages. The automaton was slightly modified to accommodate the simultaneous use of both matrices. The automaton starts with 1,000 cells, as for the standard simulation, but a tag is added to each cell to indicate its type. The initial ratio was 60:40\% of type A:type B cells (Fig. 3b). When a cell divides, its type is inherited and the next cell-cycle duration is extracted from either $M_{\mathrm{A}}$ or $M_{\mathrm{B}}$. The number of type A or type B cells is recorded at each round of simulation. The simulation is not performed over more than $\sim 35$ population doublings because of the lack of experimental data in type A matrix $M_{\mathrm{A}}$ beyond this point.

\section{References}

1. Hayflick, L. The limited in vitro lifetime of human diploid cell strains. Exp. Cell Res. 37, 614-636 (1965).

2. Harley, C. B., Futcher, A. B. \& Greider, C. W. Telomeres shorten during ageing of human fibroblasts. Nature 345, 458-460 (1990).

3. d'Adda di Fagagna, F. et al. A DNA damage checkpoint response in telomereinitiated senescence. Nature 426, 194-198 (2003).

4. Campisi, J. \& d'Adda di Fagagna, F. Cellular senescence: when bad things happen to good cells. Nat. Rev. Mol. Cell Biol. 8, 729-740 (2007).

5. Lundblad, V. \& Szostak, J. W. A mutant with a defect in telomere elongation leads to senescence in yeast. Cell 57, 633-643 (1989).

6. Teixeira, M. T. Saccharomyces cerevisiae as a model to study replicative senescence triggered by telomere shortening. Front. Oncol. 3, 101 (2013).

7. Lundblad, V. \& Blackburn, E. H. An alternative pathway for yeast telomere maintenance rescues est1- senescence. Cell 73, 347-360 (1993).

8. Shay, J. W., Reddel, R. R. \& Wright, W. E. Cancer. Cancer and telomeres--an ALTernative to telomerase. Science 336, 1388-1390 (2012).

9. Gunes, C. \& Rudolph, K. L. The role of telomeres in stem cells and cancer. Cell 152, 390-393 (2013).

10. Xu, Z., Duc, K. D., Holcman, D. \& Teixeira, M. T. The length of the shortest telomere as the major determinant of the onset of replicative senescence. Genetics 194, 847-857 (2013).

11. Abdallah, P. et al. A two-step model for senescence triggered by a single critically short telomere. Nat. Cell Biol. 11, 988-993 (2009).

12. Soudet, J., Jolivet, P. \& Teixeira, M. T. Elucidation of the DNA end-replication problem in Saccharomyces cerevisiae. Mol. Cell 53, 954-964 (2014).

13. Smith, J. R. \& Whitney, R. G. Intraclonal variation in proliferative potential of human diploid fibroblasts: stochastic mechanism for cellular aging. Science 207, 82-84 (1980).

14. Enomoto, S., Glowczewski, L. \& Berman, J. MEC3, MEC1, and DDC2 are essential components of a telomere checkpoint pathway required for cell cycle arrest during senescence in Saccharomyces cerevisiae. Mol. Biol. Cell 13, 2626-2638 (2002).

15. Ijpma, A. S. \& Greider, C. W. Short telomeres induce a DNA damage response in Saccharomyces cerevisiae. Mol. Biol. Cell 14, 987-1001 (2003).

16. Fehrmann, S. et al. Aging yeast cells undergo a sharp entry into senescence unrelated to the loss of mitochondrial membrane potential. Cell Rep. 5, 1589-1599 (2013).

17. Mortimer, R. K. \& Johnston, J. R. Life span of individual yeast cells. Nature 183, 1751-1752 (1959).

18. Voth, W. P., Olsen, A. E., Sbia, M., Freedman, K. H. \& Stillman, D. J. ACE2, CBK1, and BUD4 in budding and cell separation. Eukaryot. Cell 4, 1018-1028 (2005).

19. Lebel, C. et al. Telomere maintenance and survival in Saccharomyces cerevisiae in the absence of telomerase and RAD52. Genetics 182, 671-684 (2009).

20. Low, K. C. \& Tergaonkar, V. Telomerase: central regulator of all of the hallmarks of cancer. Trends Biochem. Sci. 38, 426-34 (2013).

21. Lingner, J. et al. Reverse transcriptase motifs in the catalytic subunit of telomerase. Science 276, 561-567 (1997).

22. Box, G. E. P., Jenkins, G. M. \& Reinsel, G. C. Time Series Analysis: Forecasting and Control (Wiley, 2008).

23. Zhao, X., Muller, E. G. \& Rothstein, R. A suppressor of two essential checkpoint genes identifies a novel protein that negatively affects dNTP pools. Mol. Cell 2 , 329-340 (1998).

24. Toczyski, D. P., Galgoczy, D. J. \& Hartwell, L. H. CDC5 and CKII control adaptation to the yeast DNA damage checkpoint. Cell 90, 1097-1106 (1997).

25. Fallet, E. et al. Length-dependent processing of telomeres in the absence of telomerase. Nucleic Acids Res. 42, 3648-3665 (2014). 
26. Le, S., Moore, J. K., Haber, J. E. \& Greider, C. W. RAD50 and RAD51 define two pathways that collaborate to maintain telomeres in the absence of telomerase. Genetics 152, 143-152 (1999).

27. Lendvay, T. S., Morris, D. K., Sah, J., Balasubramanian, B. \& Lundblad, V. Senescence mutants of Saccharomyces cerevisiae with a defect in telomere replication identify three additional EST genes. Genetics 144, 1399-1412 (1996).

28. Meyer, D. H. \& Bailis, A. M. Telomerase deficiency affects the formation of chromosomal translocations by homologous recombination in Saccharomyces cerevisiae. PLoS ONE 3, e3318 (2008).

29. Kolodner, R. D., Putnam, C. D. \& Myung, K. Maintenance of genome stability in Saccharomyces cerevisiae. Science 297, 552-557 (2002).

30. Khadaroo, B. et al. The DNA damage response at eroded telomeres and tethering to the nuclear pore complex. Nat. Cell Biol. 11, 980-987 (2009).

31. Chang, M., Arneric, M. \& Lingner, J. Telomerase repeat addition processivity is increased at critically short telomeres in a Tel1-dependent manner in Saccharomyces cerevisiae. Genes Dev. 21, 2485-2494 (2007).

32. Lydeard, J. R., Jain, S., Yamaguchi, M. \& Haber, J. E. Break-induced replication and telomerase-independent telomere maintenance require Pol32. Nature 448, 820-823 (2007).

33. Hackett, J. A., Feldser, D. M. \& Greider, C. W. Telomere dysfunction increases mutation rate and genomic instability. Cell 106, 275-286 (2001).

34. Hackett, J. A. \& Greider, C. W. End resection initiates genomic instability in the absence of telomerase. Mol. Cell Biol. 23, 8450-8461 (2003).

35. Churikov, D., Charifi, F., Simon, M. N. \& Geli, V. Rad59-facilitated acquisition of y' elements by short telomeres delays the onset of senescence. PLoS Genet. 10, e1004736 (2014).

36. Sandell, L. L. \& Zakian, V. A. Loss of a yeast telomere: arrest, recovery, and chromosome loss. Cell 75, 729-739 (1993).

37. Larrivee, M. \& Wellinger, R. J. Telomerase- and capping-independent yeast survivors with alternate telomere states. Nat. Cell Biol. 8, 741-747 (2006).

38. Payen, C., Koszul, R., Dujon, B. \& Fischer, G. Segmental duplications arise from Pol32-dependent repair of broken forks through two alternative replication-based mechanisms. PLoS Genet. 4, e1000175 (2008).

39. Vasan, S., Deem, A., Ramakrishnan, S., Argueso, J. L. \& Malkova, A. Cascades of genetic instability resulting from compromised break-induced replication. PLoS Genet. 10, e1004119 (2014).

40. Costantino, L. et al. Break-induced replication repair of damaged forks induces genomic duplications in human cells. Science 343, 88-91 (2014).

41. Branzei, D. \& Foiani, M. Maintaining genome stability at the replication fork. Nat. Rev. Mol. Cell Biol. 11, 208-219 (2010).

42. Gonzalez-Prieto, R., Munoz-Cabello, A. M., Cabello-Lobato, M. J. \& Prado, F. Rad51 replication fork recruitment is required for DNA damage tolerance. EMBO J. 32, 1307-1321 (2013)

43. Ivessa, A. S., Zhou, J. Q., Schulz, V. P., Monson, E. K. \& Zakian, V. A. Saccharomyces Rrm3p, a 5' to 3' DNA helicase that promotes replication fork progression through telomeric and subtelomeric DNA. Genes Dev. 16, 1383-1396 (2002).

44. Lee, J. Y., Kozak, M., Martin, J. D., Pennock, E. \& Johnson, F. B. Evidence that RecQ helicase slows senescence by resolving recombining telomeres. PLoS Biol. 5, 1334-1344 (2007).

45. Gao, H., Moss, D. L., Parke, C., Tatum, D. \& Lustig, A. J. The Ctf18RFC clamp loader is essential for telomere stability in telomerase-negative and mre11 mutant alleles. PLoS ONE 9, e88633 (2014).
46. Makovets, S., Herskowitz, I. \& Blackburn, E. H. Anatomy and dynamics of DNA replication fork movement in yeast telomeric regions. Mol. Cell Biol. 24, 4019-4031 (2004).

47. Deem, A. et al. Break-induced replication is highly inaccurate. PLoS Biol. 9, e1000594 (2011).

48. Huang, M. E., Rio, A. G., Galibert, M. D. \& Galibert, F. Pol32, a subunit of Saccharomyces cerevisiae DNA polymerase delta, suppresses genomic deletions and is involved in the mutagenic bypass pathway. Genetics 160, 1409-1422 (2002).

49. Smith, C. E., Lam, A. F. \& Symington, L. S. Aberrant double-strand break repair resulting in half crossovers in mutants defective for Rad51 or the DNA polymerase \{delta\} complex. Mol. Cell Biol. 29, 1432-41 (2009).

50. Romano, G. H. et al. Environmental stresses disrupt telomere length homeostasis. PLoS Genet. 9, e1003721 (2013).

\section{Acknowledgements}

We thank David Shore and Victoria Lundblad for yeast strains and plasmids; Miguel Godinho Ferreira and Claus Azzalin for discussions; and Stéphane Lemaire, Gaël Yvert, Tom Cech, Jim Haber and Joachim Lingner for critical reading of the manuscript. We also thank all members of the UMR8226 for technical support. This work was supported by the European Research Council (ERC-2010-StG 260906-D-END to M.T.T.), the Mairie de Paris (Programme Emergences to M.T.T.), ITMO Cancer and the 'Initiative d'Excellence' programme from the French State (Grant 'DYNAMO,' ANR-11-LABX-0011-01).

\section{Author contributions}

Z.X. and M.T.T. designed the project. M.T.T. oversaw the project. Z.X. performed all of the experiments, analysed the microfluidics-based time-lapse data and performed the computational and statistical analyses. Z.X., E.F., G.C. and M.T.T. interpreted and discussed the results. C.P., S.F. and G.C. developed the microfluidics-based approach and the analysis software. E.F. performed the initial experiments. Z.X., G.C. and M.T.T. wrote the paper with input from all of the authors.

\section{Additional information}

Supplementary Information accompanies this paper at http://www.nature.com/ naturecommunications

Competing financial interests: The authors declare no competing financial interests.

Reprints and permission information is available online at http://npg.nature.com/ reprintsandpermissions/

How to cite this article: $\mathrm{Xu}, \mathrm{Z}$. et al. Two routes to senescence revealed by real-time analysis of telomerase-negative single lineages. Nat. Commun. 6:7680 doi: $10.1038 /$ ncomms8680 (2015).

This work is licensed under a Creative Commons Attribution 4.0 International License. The images or other third party material in this
article are included in the article's Creative Commons license, unless indicated otherwise in the credit line; if the material is not included under the Creative Commons license, users will need to obtain permission from the license holder to reproduce the material. To view a copy of this license, visit http://creativecommons.org/licenses/by/4.0/ 